\title{
MVDC marine electrical distribution: Are we ready?
}

U. Javaid, D. Dujić, and W. van der Merwe

This material is posted here with permission of the IEEE. Such permission of the IEEE does not in any way imply IEEE endorsement of any of EPFL's products or services. Internal or personal use of this material is permitted. However, permission to reprint / republish this material for advertising or promotional purposes or for creating new collective works for resale or redistribution must be obtained from the IEEE by writing to pubs-permissions@ieee. org. By choosing to view this document, you agree to all provisions of the copyright laws protecting it. 


\section{MVDC Marine Electrical Distribution: Are we ready?}

\author{
Uzair Javaid, Dražen Dujić \\ École Polytechnique Fédérale de Lausanne - EPFL \\ Power Electronics Laboratory - PEL \\ Station 11, CH-1015, Lausanne, Switzerland \\ uzair.javaid@epfl.ch,drazen.dujic@epfl.ch
}

\author{
Wim van der Merwe \\ ABB Switzerland Ltd \\ Medium Voltage Drives \\ Austrasse, CH-5300, Turgi, Switzerland \\ wim.van-der-merwe@ch.abb.com
}

\begin{abstract}
Marine high-power on-board electrical systems are predominantly utilizing three-phase medium voltage alternating current (MVAC) distribution. Depending on the size and purpose of the ship, on-board electrical systems provide supply to loads in excess of $60 \mathrm{MW}$. Increasingly, medium voltage direct current (MVDC) distribution systems are being considered as an alternative. An increase in the fuel efficiency of the prime movers, the removal of bulky low frequency transformer and the easier integration of different storage technologies are especially attractive. This paper discusses the opportunities but also the technical difficulties associated with the transition from an MVAC to an MVDC system, using an existing LNG tanker MVAC on-board distribution system, as example.
\end{abstract}

\section{Keywords-Marine, MVDC, Distribution systems}

\section{INTRODUCTION}

Nowadays, MVAC electrical distribution systems are predominantly used for ship on-board power systems. Recent advances, in the domain of power electronics, are playing an important role in the field of electric propulsion and introduction of MVDC electrical distribution in ships. Certain activities and development work have been done already, concerning commercial ships and in particular military vessels [1]. The move towards MVDC distribution is mainly driven by the opportunity to increase the fuel-efficiency on the generation side, as well as with possibilities for more flexible designs of the complete electrical power system of a ship $[2,3]$.

With existing MVAC systems, the frequency of electrical generators has to be adjusted (kept constant) to maintain the distribution AC frequency (e.g. 50/60 Hz), irrespective of the current loading conditions. This results in a sub-optimal use of prime movers (e.g. diesel generators) and the inability to adjust their operating points more optimally [4]. Since these systems are always redundant, coordination between multiple generators is required to keep them synchronized. An MVDC distribution system offers improvements, in this regard, since due to the rectification of the generator's AC voltage; the actual operating speed can be adjusted to better match demand and supply. This flexibility offers improvements in efficiency on the generation side, but also profoundly changes the overall distribution system on the ship. It offers the possibility to use high-speed generators connected to the MVDC distribution system through rectifiers, allowing for a reduction in size, as smaller machines with higher rotational speed, lower torque and higher power density can replace the existing $50 / 60 \mathrm{~Hz}$ generators [5].
Generally, electric ship research covers a broad spectrum of fields. It has been identified as an emerging field by the IEEE technical activities board, with numerous challenges being identified. The IEEE sponsored 'Electric Ship Technologies Symposium' has been started as a bi-annual event to bring experts together and share technology developments [6]. Different standard have been formulated for power electronic equipment development and MVDC distribution in ships [7]. Some of the industrial players have already demonstrated the impacts of DC technology on a ship electrical system, although using LVDC, as described in [8]. Modeling and simulations using average models of different sub-systems of an electric ship (e.g. generators, rectifiers, propulsion units) have been presented in $[9,10]$. The overall system stability is very important for secure, continuous and uninterrupted operation and large disturbances can cause the system to become unstable. Here the presence of a large number of power converters raises concerns associated with systems operated with constant power loads, as discussed in [11]. Efficiency of an MVDC ship is evaluated in [12] providing methods for the overall system optimization.

To provide MVDC distribution, both active and passive rectifiers could be used, providing different levels of controllability over the electrical supply system [13]. Grounding strategies, such as ungrounded, high-impedance grounded, low-impedance grounded and effectively grounded have been discussed in [14] for MVDC shipboard power systems. Shipboard MVDC power systems are expected to have short cable lengths with high interconnection densities and a broad integration of power electronics components. In marine systems very high reliability and continuous service of loads is required $[15,16]$. This raises the question of protection of the DC system, as a selective and reliable protection system would be required. In [17] a coordinated strategy considering power electronic equipment and low voltage protection devices has been proposed.

Although the literature describes many advancements in the understanding of MVDC distribution in ships, there is a clear lack of industrial equipment for MVDC applications. This paper investigates some of the technical challenges in moving towards a MVDC on-board ship system. To have a reference case, an existing $\mathrm{LNG}$ tanker MVAC on-board electrical power system is considered and described in Section 2. A proposal for the possible evolution of this system into MVDC is described in Section 3. The system is discussed further in Section 4, followed by conclusions in Section 5. 


\section{REFERENCE CASE - LNG TANKER With MVAC ELECTRICAL DISTRIBUTION SYSTEM}

For better understanding of the existing systems and a proper identification of technology gaps, a real installation is considered as a reference case. The simplified single line diagram of a liquefied natural gas (LNG) tanker with an MVAC electrical distribution system is shown in Fig. 1.

The distributed generation system consists of four generators with a total installed capacity of 42 MVA and an output voltage level of $6.6 \mathrm{kV}$. The voltage for the MVAC electrical distribution system is also $6.6 \mathrm{kV}$ and is obtained directly from the generators, while the frequency of the MVAC electrical grid is $60 \mathrm{~Hz}$. A pair of generators (8.4 MVA + 12.6 MVA) is connected to each of two main switchboards (MSB 1 and MSB 2) from where different loads are supplied. The total short circuit handling capability at each of the main switchboards is $31.5 \mathrm{kA}$. In the case of loss of some generators, the two main switchboards can be interconnected through switch X.

Since the complete on-board electrical system is made redundant, for a functional description it is sufficient to consider only half of the system. With reference to MSB 1, it can be seen that there are three distinct group of loads connected to it.

The largest load on the MSB 1 is the propulsion variable speed drive $[18,19]$ for an 11 MVA, $3 \mathrm{kV}$ electrical machine connected through a gearbox to a fixed pitch propeller. Due to the machine's voltage being different from the MVAC electrical distribution voltage, two line frequency transformers (each rated at 6.4 MVA) are used at the input of a drive. This effectively provides 24-pulse rectification and voltage adaptation for the inverter stage of the variable speed drive. In addition, the complete drive system is made redundant, so that the loss of an inverter, still allows for propulsion albeit at reduced power.

The second large group of loads connected to MSB 1 consists of a bow thruster rated at $2.5 \mathrm{MW}$ and two motors rated at $0.46 \mathrm{MW}$ each. In addition, there is a $2.8 \mathrm{MW}$ rated step-down transformer with secondary voltage of $450 \mathrm{~V}$. The total installed power of this group of loads is $6.22 \mathrm{MW}$.

The third large group of loads is connected to a dedicated switchboard SB 1 (which is directly connected to MSB 1) and consists of a $1 \mathrm{MW}$ rated step down transformer, also to $450 \mathrm{~V}$, and group of 6 motors with a combined total power of 3.95 MW. The total installed power of this group of loads is 4.95 MW. The interconnecting switch $\mathrm{Y}$ allow for SB 1 to be connected to SB 2, in case of loss of a supply on MSB 1. An identical architecture is present in the left hand side of the installation as shown in Fig. 1.

From the single line diagram of Fig. 1, a few observations can be made. It can be seen that the LNG tanker system has a large number of direct-to-line high power motors directly supplied from the MVAC $6.6 \mathrm{kV}$ distribution system. The complete replacement of MVAC distribution with MVDC distribution is actually questionable in this arrangement, since the second group and third group of loads require a fixed 6.6 $\mathrm{kV} \mathrm{ac} \mathrm{voltage} \mathrm{and} \mathrm{is} \mathrm{designed} \mathrm{to} \mathrm{operate} \mathrm{without} \mathrm{any}$ converters. It would be possible to supply these loads from a dedicated converter, considering that the overall ratings are not that high. However, due to multiple parallel-connected motors with non-related operating cycles, large inrush currents would have to be tolerated, up to 6-8 times the rated current. Since this would result in a significant oversizing of the converter this solution might not be feasible. The inclusion of dedicated soft-starter(s) is another possibility, but also adds an unnecessary complication to the system.
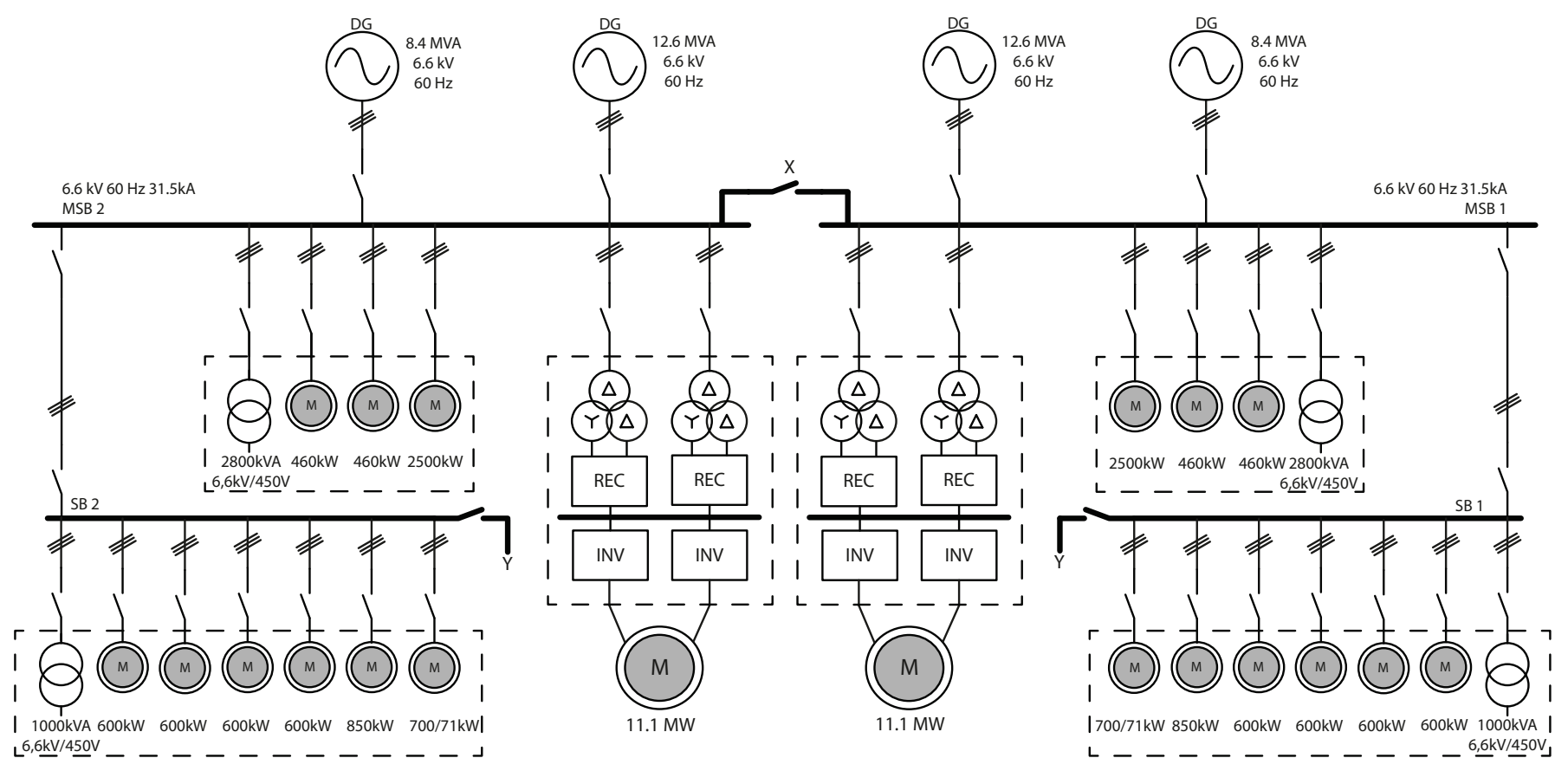

Fig. 1. Simplified single-line diagram of a MVAC electrical distribution system of a LNG tanker. Redundancy concept allows for interconnections between different switchboards using switches $\mathrm{X}$ and $\mathrm{Y}$. 
In addition to the high power loads described above, there is also a need to provide a low voltage (LV) AC supply for various hotel loads. Dedicated line frequency transformers are used for this purpose to lower the voltage to $450 \mathrm{~V}$ for further distribution throughout the ship.

Regarding the load profiles, LNG tankers spend most of their time on voyage between different stations. This accounts for $90 \%$ of operational time while the remaining $10 \%$ is used for passing through transit canals, loading and unloading LNG and other low speed tasks. During the voyage, the propulsion system works at $80 \%$ (based on average speed) of its maximum capacity or in special cases where tanker has to make up for lost time the utilization might be up to $90 \%$ capacity. Nevertheless, all other loads must have electrical power available on their respective switchboards.

\section{MVDC ELECTRICAL DISTRIBUTION SYSTEMS}

In a MVDC distribution system, power exchange between the source and loads is realized with DC power. Significant improvements on the system level are expected through better fuel efficiency on the generation side (no need to synchronize to the grid), as well as through volume/weight savings through the removal of low frequency transformers associated with various parts of the system (e.g. propulsion drive transformers). However, all loads connected to the MVDC electrical distribution will have to be modified or adapted to accommodate DC power transfer. IEEE recommendations [13] propose various voltage levels to be deployed for MVDC distribution, as indicated in Table 1. Yet, these voltage levels have to be coordinated with the voltage levels of AC generators as well as with those of AC machines, used in large numbers in the ship. This voltage coordination is not trivial, as it requires availability of power converters with voltage ratings that can vary greatly.

With an MVDC electrical distribution system, DC protection becomes the main protection scheme for the complete system, and with the voltage levels indicated in Table 1 ; the lack of available and suitable protection equipment is obvious. For low voltage loads (e.g. hotel loads), irrespective of AC or DC, the need to step-down the voltage from MVDC level to LVAC or LVDC level exists. This function that was usually performed with a line frequency transformer now has to be performed by means of some sort of power electronics converter. High power DC-DC converters are not yet a widely available industrial technology, despite large research efforts directed in this area, also including the, so called, power electronic transformer [20].

The migration from the existing MVAC system to the new MVDC systems is not straightforward task and the various recommendations [14] are not easy to apply to every ship onboard distribution system, as it will be seen from sub-sequent sections. Nevertheless, in order to explore the potential architectural evolution towards MVDC, two possible system architectures are presented. First, a partial introduction of the MVDC for the propulsion is proposed while keeping the MVAC for the remaining loads. This is referred to here as a hybrid MVAC/MVDC system. The second proposal considers a complete MVDC system, thereby completely replacing MVAC throughout the whole LNG tanker.
TABLE I. MVDC RECOMMENDED Voltage LeVELS [14]

\begin{tabular}{cccc}
\hline \hline & $\begin{array}{c}\text { MVDC } \\
\mathrm{kV}\end{array}$ & $\begin{array}{c}\text { Nominal MVDC } \\
\mathrm{kV}\end{array}$ & $\begin{array}{c}\text { Maximum MVDC } \\
\mathrm{kV}\end{array}$ \\
\hline \multirow{2}{*}{ Established } & 1.5 & $1.5( \pm 0.75)$ & $2( \pm 1)$ \\
& 3 & $3( \pm 1.5)$ & $5( \pm 2.5)$ \\
\cline { 2 - 4 } & 6 & $6( \pm 3)$ & $10( \pm 5)$ \\
Future & 12 & $12( \pm 6)$ & $16( \pm 8)$ \\
design & 18 & $18( \pm 9)$ & $22( \pm 11)$ \\
classes & 24 & $24( \pm 12)$ & $34( \pm 17)$ \\
& 30 & $30( \pm 15)$ & \\
\hline \hline
\end{tabular}

\section{A. Hybrid MVAC/MVDC System}

Considering that a large group of loads operate directly from the MVAC electrical distribution system and requires no converters for regulation, the hybrid system, as shown in Fig. 2 , preserves this architecture without modifications. Therefore, MVAC distribution is provided directly from the DG sets, and serves the direct-to-line motors (e.g. pumps, bow thrusters) and can be further step-down towards the LVAC loads using linefrequency transformers. In this configuration no optimization of the DG operating points are possible and the associated fuel saving benefits are not possible.

However, to remove the bulky line frequency transformers associated with propulsion, an MVDC electrical distribution system is introduced for this part. MVDC electrical distribution is achieved through rectification from MVAC. Due to the absence of multi-winding transformers the rectifiers (or plurality of those) are, by necessity, 6-pulse, and the MVDC distribution voltage level is defined based on actual the MVAC voltage. With passive rectification and a $6.6 \mathrm{kV}$ MVAC system, the actual MVDC level is around $10 \mathrm{kV}$. Both passive and active rectification is possible. Often it is impossible to regenerate energy from the propulsion motors or situations where this would be needed are rare (e.g. crash stop). For these reasons, braking choppers and associated braking resistors are normally used, even though this is not directly indicated in Fig.1. In a hybrid system with other loads being actively connected to the MVAC system, eventual regeneration would be possible through active rectifiers, removing a need for braking choppers/resistors.

Propulsion inverters are connected directly to the MVDC, as indicated in Fig. 2. However, both inverters and propulsion motors from Fig. 2 are not the same as those in original system from Fig. 1. The motor rated voltage has to match the MVDC distribution voltage, and in this case it would have to be doubled resulting in a $6 \mathrm{kV}$ propulsion motor. Equally, the high power inverter must operate directly from $10 \mathrm{kV}$ (this is again different from Fig. 1 where the DC bus voltage was $5 \mathrm{kV}$ ). This implicates major change in the propulsion chain.

From a system point of view, the introduction of MVDC electrical distribution allow for spatial optimization of the propulsion system, considering that rectifiers and inverters do not have to be located close to each other. This together with the removal of the input transformers offers certain degrees of freedom for the ship designers. The redundancy concept is preserved and expanded to MVDC section of a system as well, and interconnection of DC SB 1 and DC SB 2 is possible through the switch $\mathrm{Z}$ (e.g. to bypass a broken rectifier). 


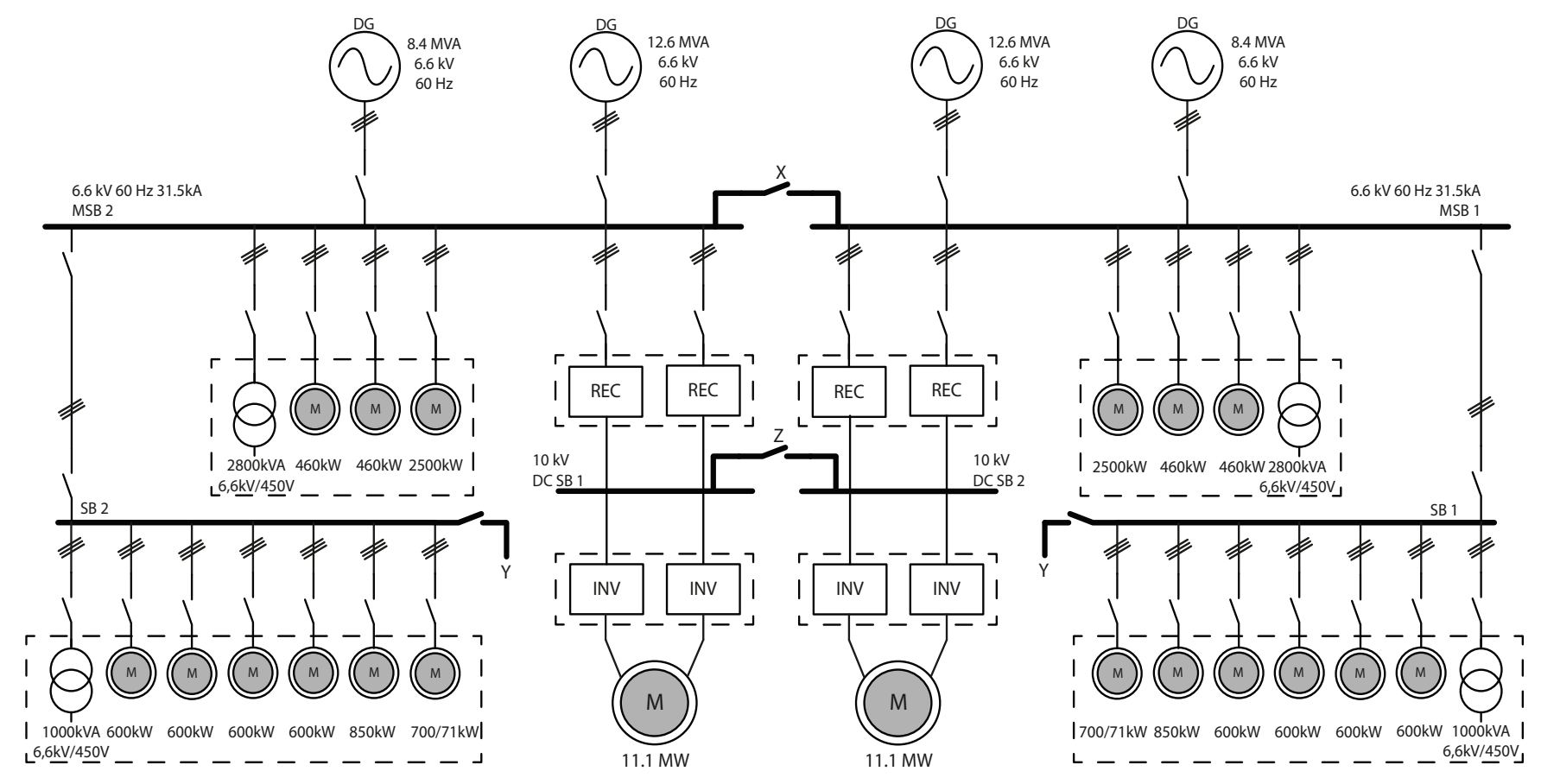

Fig. 2. Single-line diagram of proposed hybrid MVAC/MVDC electrical distribution system of a LNG tanker. Propulsion inverters and propulsion motors voltage ratings are double of those from Fig.1.

\section{B. Full MVDC System}

Instead of a hybrid system, a complete makeover into a full MVDC system is proposed here, as shown in Fig. 3. The MVDC system is obtained through a dedicated rectifier associated with each DG unit. Again, the MVDC level is directly related to the chosen generator voltage level, and for an LNG tanker it would result in around $10 \mathrm{kV}$ DC. It would be advantageous to use multi-phase generators (e.g. multiple of three-phase windings) instead of the three-phase DGs from Fig.1, so that rectification with higher pulse numbers can be applied. Passive or active rectifiers could be used equally, depending on the overall control concept deployed on the ship. The efficient use of DGs is now possible and operating speed can be adjusted based on power demand.

The MVDC electrical distribution system is distributed throughout the ship and made available on dedicated switchboards. All propulsion inverters are connected directly to MSB 1 or MSB 2, and located close to their respective loads.

The introduction of a MVDC electrical distribution system raises issues with the supply of a large group of loads that do not require converters (e.g. direct-to-line motors) and were previously operated directly from MVAC supply. As discussed earlier, this aspect needs to be addressed in a way that is efficient, simple and cost effective. The system depicted in Fig. 3 assumes the use of an adequately rated inverter for each electrical machine. The voltage class of these machines is preserved as in the original system from Fig.1, and with inverters output voltage of sufficient quality (e.g. sine filter at output), the same direct-to-line machines can be used. The cost of a system is greatly increased, but controllability and efficiency are improved as well, under the assumption that the motors do not run constantly at full power.

Equal considerations are applied with respect to hotel loads, where a dedicated inverter and transformer are used to provide $450 \mathrm{~V}$. Alternatively, LVAC could be obtained from MVDC by means of dedicated high power step-down DC-DC converter followed by an inverter. Yet, there is a certain lack of technologies that make this choice difficult at the time, especially considering large voltage ratio resulting in a need to use medium frequency transformers for galvanic isolation. Redundancy by means of interconnects switches $\mathrm{X}$ and $\mathrm{Y}$ is implemented, similarly to the original system.

The protection concept now has to consider MVDC and the handling of any faults appearing directly before or after the switchboards. The use of converters for fault current limiting functionality, depends heavily on the actual topology being used as well as on type of semiconductors. The lack of a suitable and standardized MVDC breaker technology for the considered DC voltages makes it hard to properly assess the required $R \& D$ effort in this area.

\section{TECHNOLOGY GAPS}

From the previous discussion, it is clear that MVDC electrical distribution systems, despite their attractiveness, nowadays are not supported with availability of suitable industrial equipment. This lack of technology is visible in different parts of the system. In MVDC electrical distribution systems the voltage directly defines the required voltage of the electrical generators, as well as the rated voltage of the electrical machines used on the load side. In MVAC systems this is not necessarily the case and these voltages may be independently selected. 


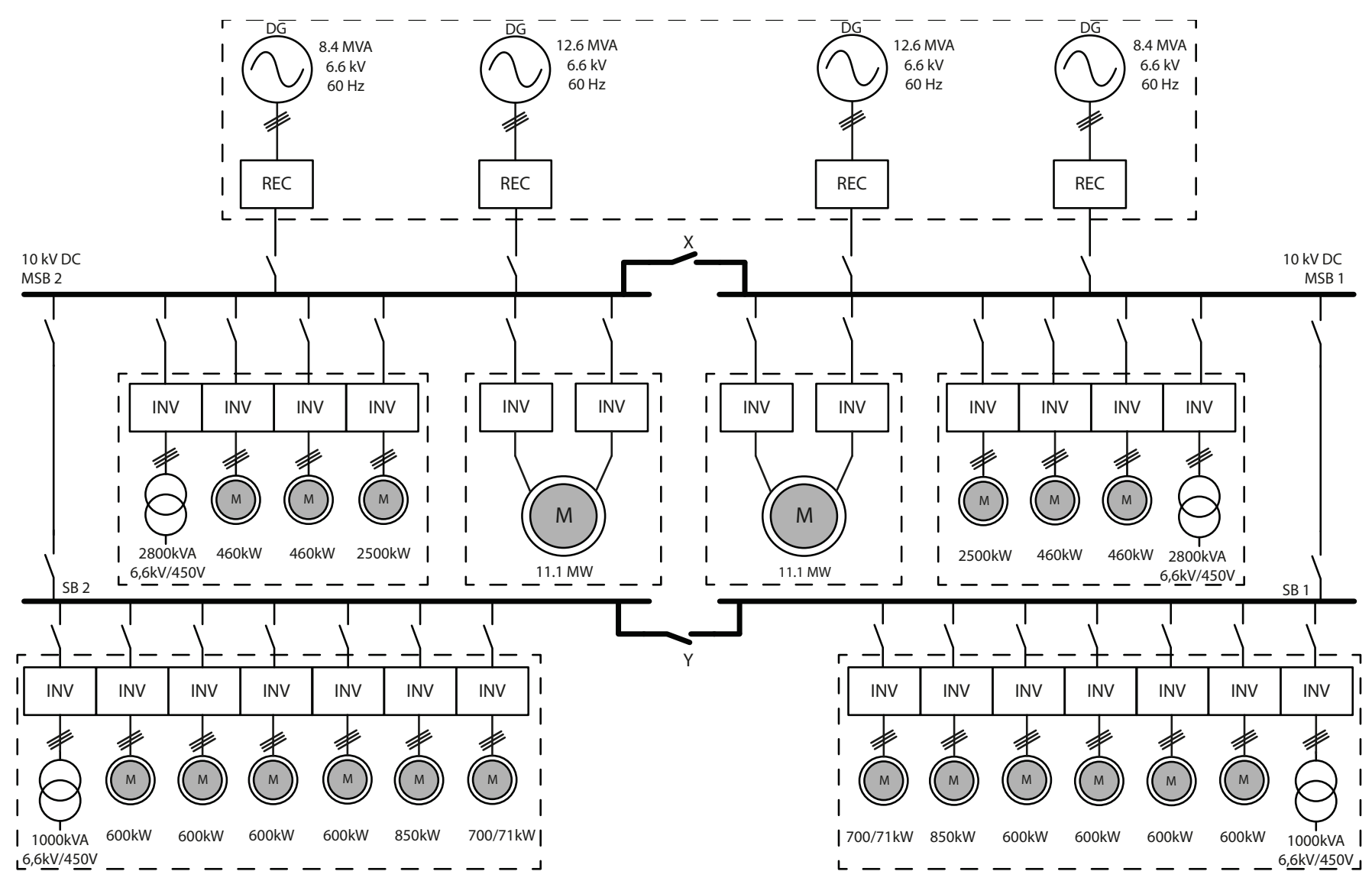

Fig. 3. Single-line diagram of proposed full MVDC electrical distribution system of a LNG tanker.

Furthermore, in the MVAC system, the electrical generators are predominantly 3-phase systems while for a MVDC system it would be more advantageous to use generators with more than 3 phases. The number of phases of generator has a direct relation to the rectifier configuration of the system. Usually three phase generators are employed but generators with more phases (multiples of three e.g. 6, 9, 12, 18) can be directly connected to passive multi-pulse rectifiers. This improves the quality of rectified MVDC electrical distribution voltage, lowers the per-phase current ratings and improves the reliability at the level of generators (redundancy through multiple set of three-phase windings). The use of active rectifiers allow for wider speed operational range of generators and improved MVDC control, even though regeneration from MVDC is not needed. Filtering at the output of rectifiers would have to be properly designed considering loading profiles as well as overall power management system on the ship.

MVDC cables are another technology that will have to be standardized for ship applications. At the moment MVAC cables are available but no commercial MVDC tailored cables can be found. The overall stability and interactions between different elements of the system have to be analyzed during the system design phase. Passive and active filters may play a role in the MVDC distribution, and at the moment there are no commercial solutions available, and considering large range of possible voltages (Tab. 1), customization may be needed.
High power inverters, placed between the MVDC system and AC loads, would require the use of capacitor banks at the inverter terminals impacting overall system stability. Proper design of these is essential for stable and robust operation of the drive but also has impact in case of faults as it can increase fault currents to unmanageable levels. Therefore, their design requires evaluation of numerous aspects.

Proposed MVDC voltage levels require suitable inverter topologies able to operate from such a high DC links. There are not many (if any) commercially available high performance drives for MVDC voltage above $10 \mathrm{kV}$. Various multilevel topologies are possible candidates, based either on series connection of semiconductor devices or multiple converter stages.

Propulsion motors make up for more than 50\% of the electrical load on ships. There are different possible motors that can be used as propulsion/thruster motors. The most popular choice is induction motor because of its rugged design and minimum maintenance requirements. However, both permanent magnet synchronous motor (PMSM) and wound rotor SM are in use due to their high efficiency and dynamic performance. With the new voltage levels (Table 1), motor voltage would need to be selected to match the system voltage level. This also implies an increase from commonly used machine voltage classes in MVAC system. Additionally, insulation coordination must consider increased stresses, due to supply provided from power electronic converters. 
As a short summary, Table 2 and Table 3 provide short overview of possible benefits and challenges associated with two proposed architectures. From the tables we can see that the biggest hurdle in implementation and realization of the MVDC system is the lack of commercial availability of high power MVDC equipment. This can be identified throughout the whole propulsion chain, from generators to electrical motors.

TABLE II. POTENTIAL BENEFITS AND CHALLENGES OF A HYBRID MVAC/MVDC ELECTRICAL DISTRIBUTION SYSTEM

\begin{tabular}{|l|l|}
\hline \multicolumn{1}{|c|}{ Potential benefits } & \multicolumn{1}{|c|}{ Challenges } \\
\hline $\begin{array}{l}\text { Less invasive ship architecture } \\
\text { modification }\end{array}$ & $\begin{array}{l}\text { Fixed speed prime movers and } \\
\text { generators }\end{array}$ \\
\hline $\begin{array}{l}\text { Removal of bulky propulsion drives } \\
\text { transformers }\end{array}$ & $\begin{array}{l}\text { Inefficient use of direct-to-line } \\
\text { motors (as before) }\end{array}$ \\
\hline $\begin{array}{l}\text { Possibility to re-generate from } \\
\text { MVDC to MVAC }\end{array}$ & $\begin{array}{l}\text { Two different protection schemes } \\
\text { (AC and DC) }\end{array}$ \\
\hline $\begin{array}{l}\text { Possibility to exchange energy } \\
\text { between drives }\end{array}$ & $\begin{array}{l}\text { Non-availability of high power DC- } \\
\text { DC converters }\end{array}$ \\
\hline AC protection is already available & $\begin{array}{l}\text { Voltage coordination in not flexible } \\
\text { MVDC, MVAC }\end{array}$ \\
\hline
\end{tabular}

TABLE III. POTENTIAL BENEFITS AND CHALLENGES OF A FULL MVDC ELECTRICAL DISTRIBUTION SYSTEM

\begin{tabular}{|l|l|}
\hline \multicolumn{1}{|c|}{ Potential benefits } & \multicolumn{1}{|c|}{ Challenges } \\
\hline $\begin{array}{l}\text { High speed prime movers and } \\
\text { generators }\end{array}$ & $\begin{array}{l}\text { No commercial rectifiers for } \\
\text { MVDC }\end{array}$ \\
\hline $\begin{array}{l}\text { Removal of bulky propulsion drives } \\
\text { transformers }\end{array}$ & Lack of MVDC protection devices \\
\hline $\begin{array}{l}\text { Possibility to re-generate from } \\
\text { MVDC }\end{array}$ & $\begin{array}{l}\text { Two different protection schemes } \\
\text { for LVAC and MVDC }\end{array}$ \\
\hline $\begin{array}{l}\text { Possibility to exchange energy } \\
\text { between drives }\end{array}$ & $\begin{array}{l}\text { Non-availability of high power DC- } \\
\text { DC converters }\end{array}$ \\
\hline $\begin{array}{l}\text { Ability to integrate energy storage } \\
\text { into MVDC }\end{array}$ & $\begin{array}{l}\text { Voltage coordination and lack of } \\
\text { DC-DC high power converters }\end{array}$ \\
\hline
\end{tabular}

\section{CONCLUSION}

MVDC electrical distribution has been considered as way forward for ship on-board electrical systems. However, there is a need for much developmental work in this domain due to a lack of industrial equipment. This analysis has considered an LNG tanker with MVAC system and its evolution towards two possible MVDC systems were presented. Both hybrid and full MVDC systems have their own challenges, and the simple transformation of an MVAC system to MVDC is not easily done. For MVDC electrical distribution systems to become reality, further developments are needed in the area of medium voltage drives, high voltage electrical machines, high power DC-DC converters, rectifiers, MVDC protection components, MVDC cable technology, application and design of energy storage systems, active and passive filters, diesel engines and gas turbines. All of these technologies would have to be fully developed before MVDC electrical distribution systems can become a reality, and truly offer advantage over the MVAC system.

\section{REFERENCES}

[1] T.J. McCoy, "Trends in ship electric propulsion," in Proc. of Power Engg. Society Summer Meeting, 2002, vol. 1, pp. 343-346.

[2] H. Holttinen et al., "Currents of change," IEEE Mag. Power and Energy, vol. 9, no. 6, pp. 47-59, Nov-Dec 2011.

[3] A. Tessarolo, S. Castellan, R. Menis, and G. Sulligoi, "Electric generation technologies for all-electric ships with Medium-Voltage DC power distribution systems," in Electric Ship Tech. Symp. (ESTS), 2013, pp. 275-281.

[4] B. Zahedi, L.E. Norum, and K.B. Ludvigsen, "Optimization of fuel consumption in shipboard power systems," in Proc. of IECON 2013, pp. 1124-1129.

[5] R.M. Calfo, J. A. Fulmer, and J. E. Tessaro, "Generators for use in electric marine ship propulsion systems," in Proc. of Power Engg. Society Summer Meeting, 2002, pp. 254-259.

[6] Y. Khersonsky, "New IEEE standards for ships," in Electric Ship Tech. Symp. (ESTS), 2011, pp. 424-429.

[7] Y. Khersonsky, "New IEEE Power Electronics Standards for Ships," in Electric Machines Tech. Symp. (EMTS) 2012.

[8] J. F. Hansen, J. O. Lindtjørn, and K. Vanska, "Onboard DC grid for enhanced DP operations in ships," in Proc. of MTS Dynamic Positioning Conf., 2011, pp. 1-8.

[9] A. Ouroua, L. Domaschk, and J.H. Beno, "Electric ship power system integration analyses through modeling and simulation," in Electric Ship Tech Symp (ESTS), 2005, pp. 70-74.

[10] B. Zahedi and L.E. Norum, "Modeling and simulation of all-electric ships with low-voltage dc hybrid power systems," IEEE Trans. Power Electron., vol. 28, no. 10, pp. 4525-4537, Oct 2013.

[11] M. Belkhayat, R. Cooley, and A. Witulski, "Large signal stability criteria for distributed systems with constant power loads," in Proc. of PESC, 1995, pp. 1333-1338.

[12] B. Zahedi and L.E. Norum, "Efficiency analysis of shipboard dc power systems," in Proc. of IECON 2013, pp. 689-694.

[13] A. Hasanzadeh, C.S. Edrington, D.M. Soto, and G.M. Rivera, "Comparative study of intensive pulse load impact on active and passive rectification system in MVDC ship power generation unit," in Proc. of IEMDC, 2013, pp. 1326-1332.

[14] IEEE, Recommended Practice for $1 \mathrm{kV}$ to $35 \mathrm{kV}$ Medium-Voltage DC Power Systems on Ships, Std. 1709-2010, vol., no., pp.1-54.

[15] B. Jacobson and J. Walker, "Grounding consisderations for dc and mixed dc and ac Power Systems," Naval Engineers Journal, vol. 119, no. 2, pp. 49-62, Oct 2007.

[16] Y. Nyanteh, L. Graber, C. Edrington, S. Srivastava, and D. Cartes, "Overview of simulation models for partial discharge and electrical treeing to determine feasibility for estimation of remaining life of machine insulation systems," in Proc. of Electrical Insulation Conf. (EIC), 2011, pp. 327-332.

[17] R.T. Wang, L.J. Fu, F. Xiao, and X.X. Fan, "System Protection for Vessel DC Zonal Electrical System Supplied by Medium Voltage DC," in Proc. of Intl. Conf. on Applied Supercond and Electromagnet Devices, 2013, pp. 89-93.

[18] J. Wahlstroem, D. Dujic, M.A. Luescher, and S. Reist, "High Power IGCT based Multilevel Inverter," in Proc. of PCIM Europe, 2014, pp. 16.

[19] D. Dujic, J. Wahlstroem, and J.A. and Fritz, D. Marrero Sosa, "Modular medium voltage drive for demanding applications," in Proc. of IPEC, 2014, pp. 3476-3481.

[20] C. Zhao et al., "Power Electronic Traction Transformer-Medium Voltage Prototype," IEEE Trans. Industrial Electron., vol. 61, no. 7, pp. 3257-3268, July 2014. 